\title{
Structural Health Monitoring of a masonry viaduct with Fibre Bragg Grating sensors
}

\author{
Haris Alexakis \\ University of Cambridge, UK
}

\author{
Andrea Franza \\ Universidad Politécnica de Madrid, Spain
}

\author{
Sinan Acikgoz \\ University of Oxford, UK
}

Matthew DeJong

University of California, Berkeley, US

Contacting author: ca510@cam.ac.uk (Haris Alexakis)

\begin{abstract}
The Marsh Lane viaduct is a masonry railway bridge constructed during the 19th century nearby Leeds Central Railway Station. The bridge appears significantly damaged due to the increase of the operational train loads over the last decades and due to environmental effects. Due to this degradation, extensive repair was conducted in 2015. After this repair work, an extensive fibre optic sensor network was installed below three spans of the bridge to monitor surface strains at 68 locations on the underside of the arch spans. The paper compares data collected from two monitoring periods, 16 months apart. Combining statistical analysis and signal processing techniques, the results show that local damage, as well as change in the global dynamic behaviour of the structure over time, can be effectively detected with the use of Fibre Bragg Grating sensors.
\end{abstract}

Keywords: ageing infrastructure, remote monitoring, fibre optics, signal processing, damage detection, masonry deterioration 


\section{Introduction}

As the population grows, an increasing number of people rely on established transport and utilities networks. In particular, ageing masonry bridges comprise $50-60 \%$ of the European rail stock $[1,2]$. The majority of these structures were built more than a century ago, before the enforcement of building codes, and their condition deteriorates with time due to the combined action of increased train loads, support movements, and environmental effects. Today, engineers are facing the challenge of maintaining these vital assets, while at the same time there is a limited capability to answer two important questions: (a) what is the current state, or performance, of the structure, and (b) how quickly is deterioration happening (Figure 1).

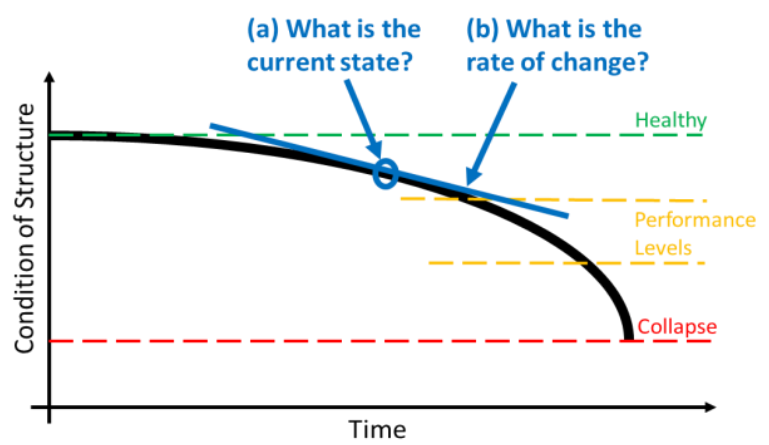

Figure 1. Typical deterioration curve of an ageing structure

The Finite Element Method (FEM), the most common choice for structural engineers to address these questions, may provide realistic results for the assessment of the existent stress fields of ageing masonry structures, with the condition that all the complex material parameters, structural arrangements, and loading conditions are well known. However, this is challenging to achieve in practice [3]. Masonry is a non-uniform, composite material, with low tensile strength and an inherently discontinuous nature. At the same time, due to the absence of monitoring data, there is no information on the deformation or loading histories of the structure.

In order to address these challenges, the Center for Smart Infrastructure and Construction (CSIC) at the University of Cambridge, UK installed a longterm Structural Health Monitoring (SHM) system with a network of Fibre Optic (FO) sensors in a damaged masonry rail bridge. The objective of this monitoring is to better understand the current serviceability state of the bridge, including the variation and magnitude of the strain distribution under typical loading, and evaluation of how the strain changes with time. Furthermore, CSIC collaborates with the Alan Turing Institute, UK and the Department of Mathematics, Imperial College London, UK to combine this technology with streaming statistical analysis for the development of real-time structural alert systems $[4,5]$.

The current study shows that over the last two years there has been a statistically significant amplification of the dynamic deformation of the bridge at specific locations, where the formation of new cracking has started. Furthermore, FO sensors appeared to be resilient and suitable for long-term monitoring of existing masonry structures.

\section{Description of the bridge}

The Marsh Lane Viaduct is located next to the Eastern entrance of Leeds Railway Station, in the centre of the city. The bridge was constructed between 1865 and 1869 [6]. Today it carries two electrified tracks with a traffic load that exceeds 200 trains per day, ranging from typical passenger trains to multi-wagon freight trains. Figure 2 shows the southern side of the investigated section of the bridge, which comprises the Arches 37,38 and 39.

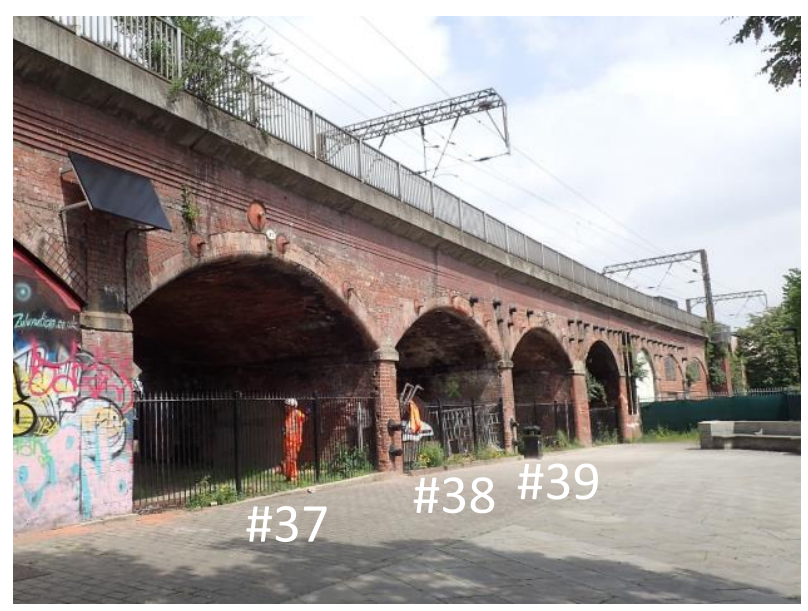

Figure 2. The southern side view of the Marsh Lane Viaduct in Leeds, UK 


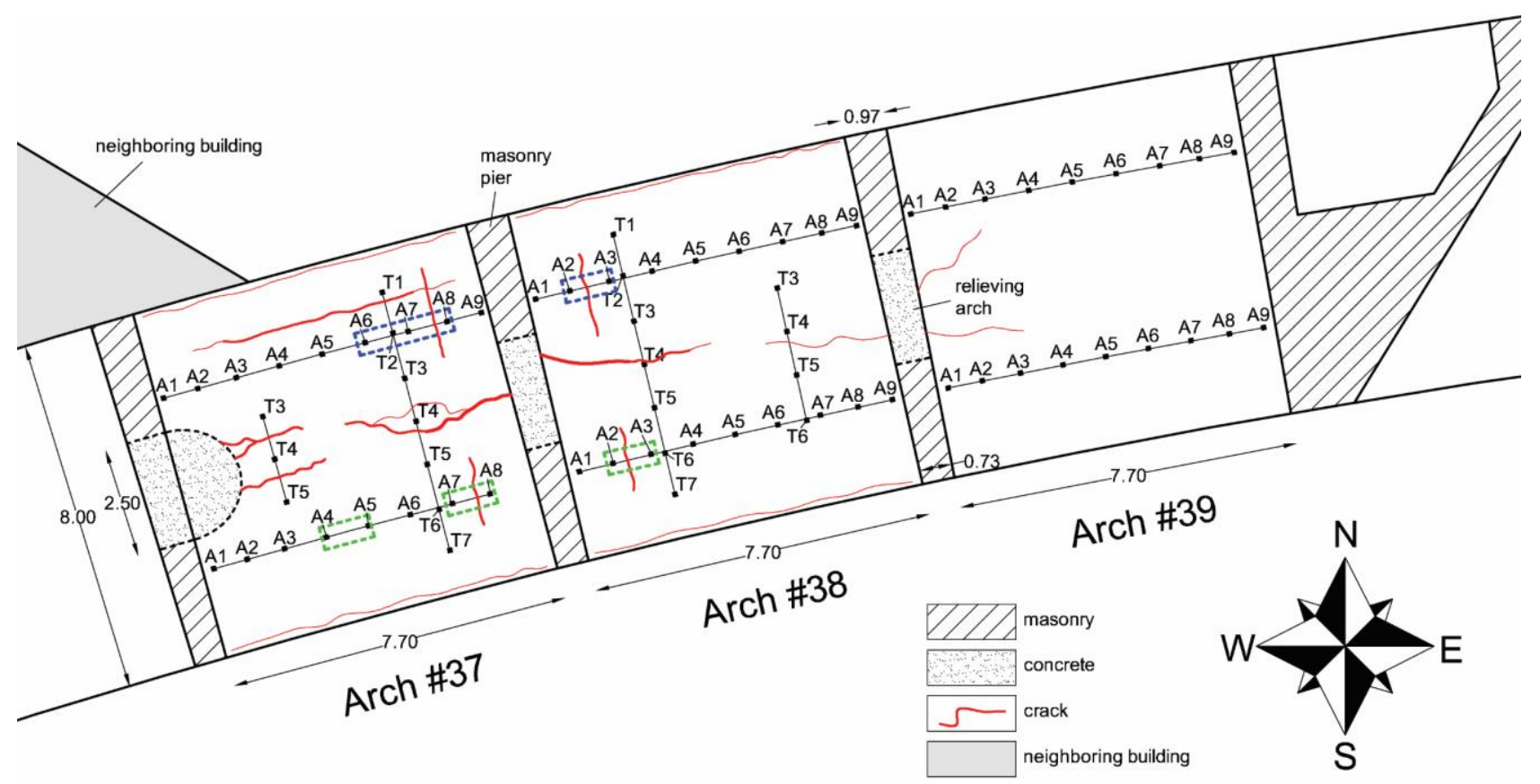

Figure 3. Plan view of the investigated section of the Marsh Lane Viaduct, showing the main damages observed under the arches (in red) and the installed fibre optic sensors network

Bridge dimensions and damages are discussed in detail in Acikgoz et al. [7]. Figure 3 shows the plan view of the bridge that summarizes the main damages in the arches. Arch 37 is the most damaged arch, followed by 38 and 39 . The most severe damages are concentrated over the relieving arches at the centre of the piers due to a spreading mechanism that forces the relieving arch keystone to descend and the walls to bow outwards [7]. For this reason, Network Rail, the owner of the bridge, conducted in 2015 an extensive repair by filling in the relieving arches with concrete and installing steel ties to arrest transverse movements of the piers and the spandrel walls, as shown in Figure 4. This mechanism is observed in both piers between Arches 37-38 and Arches 38-39, although in the first case masonry degradation is more intense. Furthermore, over the $37 / 38$ pier, there are symmetric transverse cracks in the arches at the level of the top of the backing, where the backfill level starts (see Figure 4). According to [7], these separation cracks are related to the out-of-plane rocking response mechanism of the pier. In addition, a longitudinal crack along the Arch 37 develops below the northern trail of the bridge.

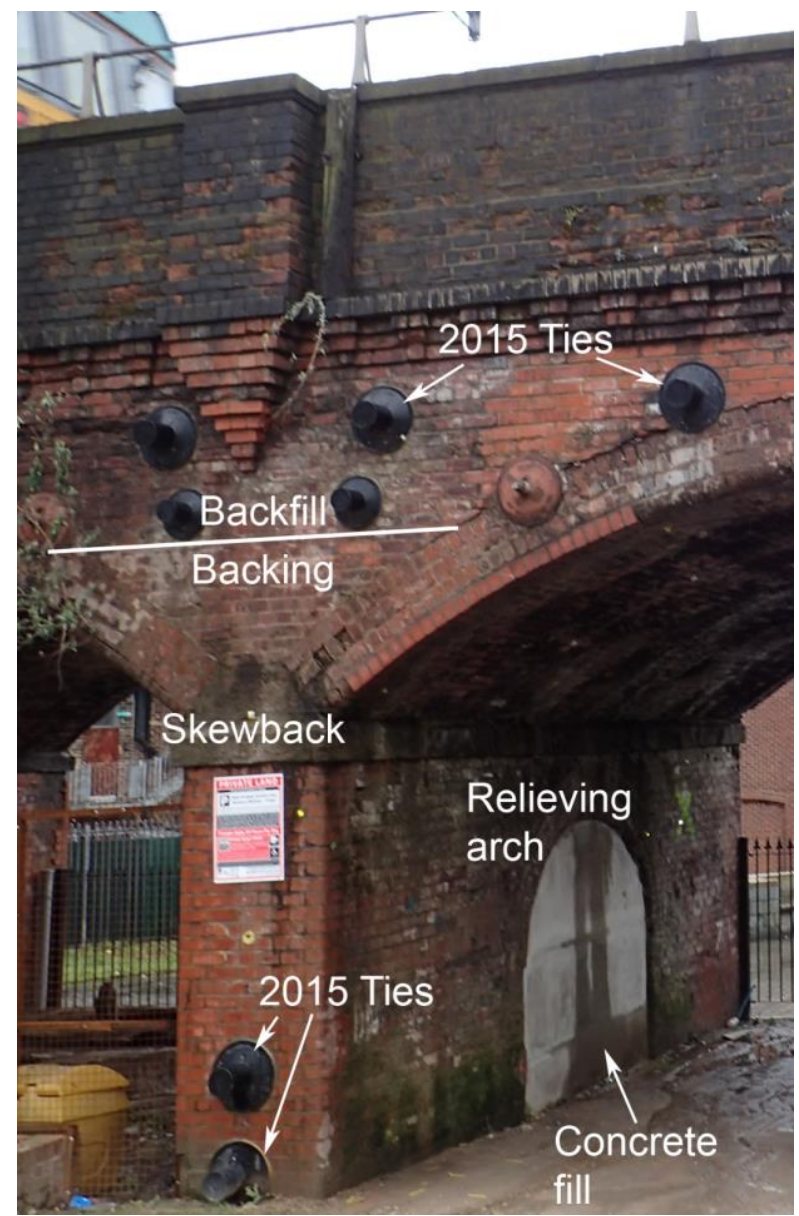

Figure 4. Northern view of the pier between Arches 38 and 39. 


\section{Sensing System}

The idea of making sensors out of optical fibres is not new and goes back at the late 70s [8]. However, it was not until the optical telecommunications boom of 2000 and the rapid expansion of the Internet that Fibre Optic (FO) sensors began to attract the interest of the scientific and industrial sectors. During the first decade of the millennium, there were limited practical applications in the field of SHM [9]. Nevertheless, over the last decade there have been several successful examples such as the monitoring of geotechnical structures, dams, pipelines, and bridges $[10,11]$.

Today, FO sensors are used to measure mechanical strain, pressure, and temperature, among other quantities, and can be divided in two main categories; quasi-distributed and distributed sensors [8]. The first category is mainly represented by Fibre Bragg Grating (FBG) sensors. In an FBG, tiny diffraction gratings inside the fibre core filter a specific wavelength of light and reflect it back to the source. The wavelength of the reflected light shifts in proportion to temperature and strain changes. In the second category of FO sensors, the whole cable works as a sensor by studying changes in the spectrum of backscattered light within the fibre. The main advantage of FO technology is that, using only a few cables, it is possible to cover large areas with multiple high performance sensors.

In order to assess the performance of the bridge and the effectiveness of the 2015 retrofitting intervention, Network Rail collaborated with CSIC in Cambridge University for the installation of an FBG sensors network underneath Arches 37 and 38. This was the first FBG deployment in a masonry rail bridge [7]. One of the objectives of this short-term installation was to test the efficiency of the system and better plan a permanent monitoring system with FBGs, which was subsequently installed in November 2017.

The long-term monitoring system shown in Figure 3 comprises:

- 47 FBG strain sensors in the longitudinal direction, at the North and South sides of Arches 37,38 and 39
- 17 FBG strain sensors in the transverse direction, at the East and West sides of Arches 37 and 38

- 4 FBG sensors attached to steel wires that connect the springings of Arch 37 and Arch 39, measuring relative horizontal displacement of the top of the piers

- 5 FBG sensors calibrated to measure temperature, distributed over different locations in the bridge.

For these 73 sensors, four custom designed arrays of FBGs were used. Each one of the four cable arrays has a Germanium doped single mode silica fibre with 20 FBG sensors distributed every one meter. The cables are fixed on the bricks using precision machined aluminium clamps, which appear as small squares in Figure 3. The clamps along the longitudinal direction are denoted with the letter A, numbered from West to East (A1-A9), and in the transverse direction with the letter $T$, numbered from North to South (T1-T7). The FBGs are located between the clamps, measuring relative strain between two fix points.

The wavelength of the backscattered light is sensitive to strain and temperature change. In this study, only the dynamic deformation of the bridge at the instance of train loading is considered, where the temperature fluctuation is marginal. In that case, the change in strain, $\Delta \varepsilon$, at the location of the FGB sensor is given by

$$
\Delta \varepsilon=\frac{\lambda-\lambda_{o}}{\lambda_{o}} \frac{1}{1-\rho},
$$

where $\lambda_{0}$ is the original transmitted wavelength, which is constant for each FBG sensor, $\lambda$ is the instant measured wavelength that changes with strain variation, and $\rho=0.23$ is the photo-elastic constant.

The 4-channel sm130 Optical Sensing Interrogator of Micron Optics, Inc. that is used in this study offers up to $1 \mathrm{kHz}$ sampling rate per sensor and 23 micro-strain $(\varepsilon \mu)$ resolution. The interrogator is continuously acquiring data, but is triggered to log only strains from train passage. The interrogator is permanently installed under Arch 37, inside the outdoor cabinet shown in Figure 5. The cabinet is equipped with 110 Watt heater and two fans controlled by thermostats, to protect the 
equipment from humidity and extreme temperatures. The interrogator is connected to a laptop with a router, which transmits remotely daily data to the CSIC server at the Department of Engineering, University of Cambridge, through a $4 \mathrm{G}$ internet connection. The data are shared with the Alan Turing Institute through an SFTP (Secure File Transfer Protocol) connection between CSIC and Department of Mathematics, Imperial College servers.

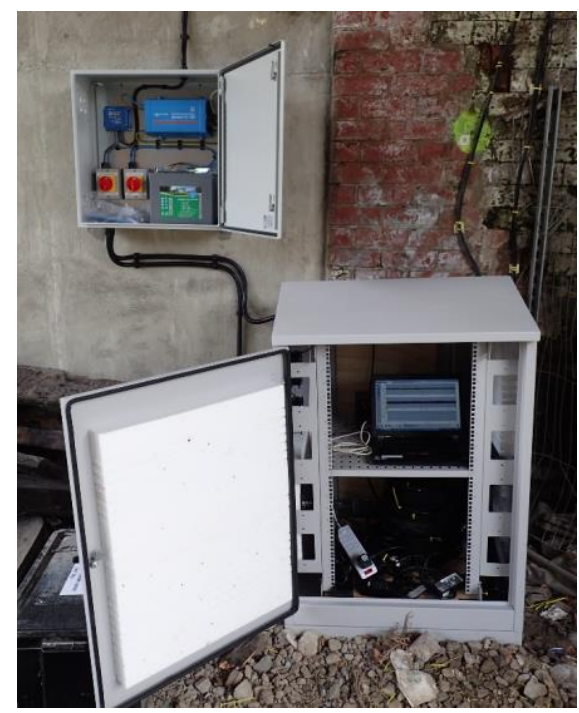

Figure 5. Current view of the data acquisition system

\section{Signal Processing}

Figure 6 presents a typical dynamic response of the sensor "37NA6A7" under train loading, meaning the longitudinal sensor between clamps A6 and A7 at the North side of Arch 37 (see Figure 3 ). The signal corresponds to the most common passenger train type, the British Rail Class 185, with three carriages and typical bogie weight 33.4 tons. Positive values correspond to relative tensile strain, and negative to relative compressive strain. The Class 185 3-carriage train represents approximately $50 \%$ of the data. Another $20 \%$ corresponds to Class 155 with 2 carriages and typical bogie weight 19.2 tons. The rest 30\% represents other passenger trains with four or more carriages (e.g. Classes 222, 144) or multiwagon freight trains.

It was noted that each train type has a specific signature signal per sensor depending on the class, number of carriages and direction. The trains heading East are using the North trail, causing the North side of the bridge to deform more, whereas the trains heading West (entering Leeds station) are using the South trail, loading more the South side of the bridge.

A code in MATLAB was developed to post-process the signals and automatically group the loading events based on train type and direction. This permits direct comparison of the dynamic response of the bridge at 68 locations for the exact same train loading over time. For instance, in Figure 6 the typical response of the 37NA6A7 sensor for the 3-carriege Class 185 train in July 2016 (red line) is compared with the response at the same location, same train, speed and direction in November 2017 (black line). Although the majority of the sensors presented identical signals, few locations, like the 37NA6A7, presented a significant amplification of the dynamic deformation.

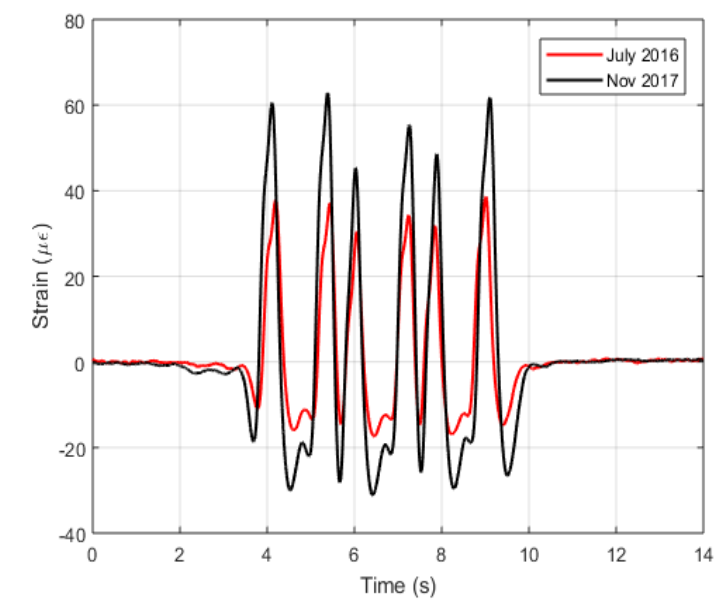

Figure 6. Change in the dynamic deformation of a damaged area of the bridge between July 2016 and November 2017

The algorithm applies cross-correlation techniques to find time lags between similar signals, for instance, signals from the same sensor location in Arches 37 and 39 (e.g. cross-correlation between keystone sensors 37NA4A5 and 39NA4A5). Given that the distance between the arches is known, the time lag corresponds to a specific train speed and direction.

The number of positive and negative peaks corresponds to a specific number of carriages and the relative distance between the positive peaks corresponds to the relative distance between train 
axles, which is characteristic for each train class. For the train direction and class identification a simple peak analysis in MATLAB is giving reliable results for the majority of the train events. A small percentage of events where the train speed over bridge was not constant (e.g. train was stopping or accelerating) or when two trains were passing over the bridge simultaneously, were separated from the typical response signals.

\section{Statistical Analysis}

This study presents results from statistical analysis of the dynamic strain variation per sensor location over time. In particular, mean value and standard deviation of the positive and negative peaks of the response for each sensor location has been calculated for 95 records in July 2016 during the first FO sensors deployment, and 62 records in November 2017, 148 records in March 2018 and 116 records in June 2018, during the second (permanent) FO sensors deployment. Performing a statistical analysis for all trains, including passenger and multi-wagon freight trains, resulted in a big dispersion of data, which does not permit for direct comparison over time. However, if the analysis is performed for a specific train type and direction, as discussed in the previous section, then the dispersion of data (standard deviation) drops significantly.
Figure 7 presents mean value and standard deviation of the maximum (positive/tensile) and minimum (negative/compressive) relative strains for 42 sensor locations that the 2016 and 2017-18 deployments had in common. The statistical analysis is performed for the Class 185 3-carriege train, heading East, which is the reason why $37 \mathrm{~N}$ and $38 \mathrm{~N}$ longitudinal sensors under the North trail recorded bigger strains compared to $37 \mathrm{~S}$ and $38 \mathrm{~S}$ longitudinal sensors under the South trail. The bold black line represents the dynamic deformation of the bridge in July 2016, whereas the green, blue and red lines corresponds to November 2017, March and June 2018.

While the response is similar in most locations, the analysis reveals that there is a significant amplification of the dynamic deformation of the bridge at the sensor locations inside the boxes with dashed blue line, shown in Figures 3 and 7, between the first and the second installation, which are more than 16 months apart. In particular there is a $100 \%$ increase in 37NA6A7, and $a 50 \%$ in 37NA7A8 and 38NA2A3. These sensors are located next to the most damaged pier between Arches 37 and 38, at the location of the transverse separation cracks. In addition, this significant increase happened in the locations that present the biggest anomaly; meaning that they deform much more compared to symmetric locations in the same or neighbouring arch.

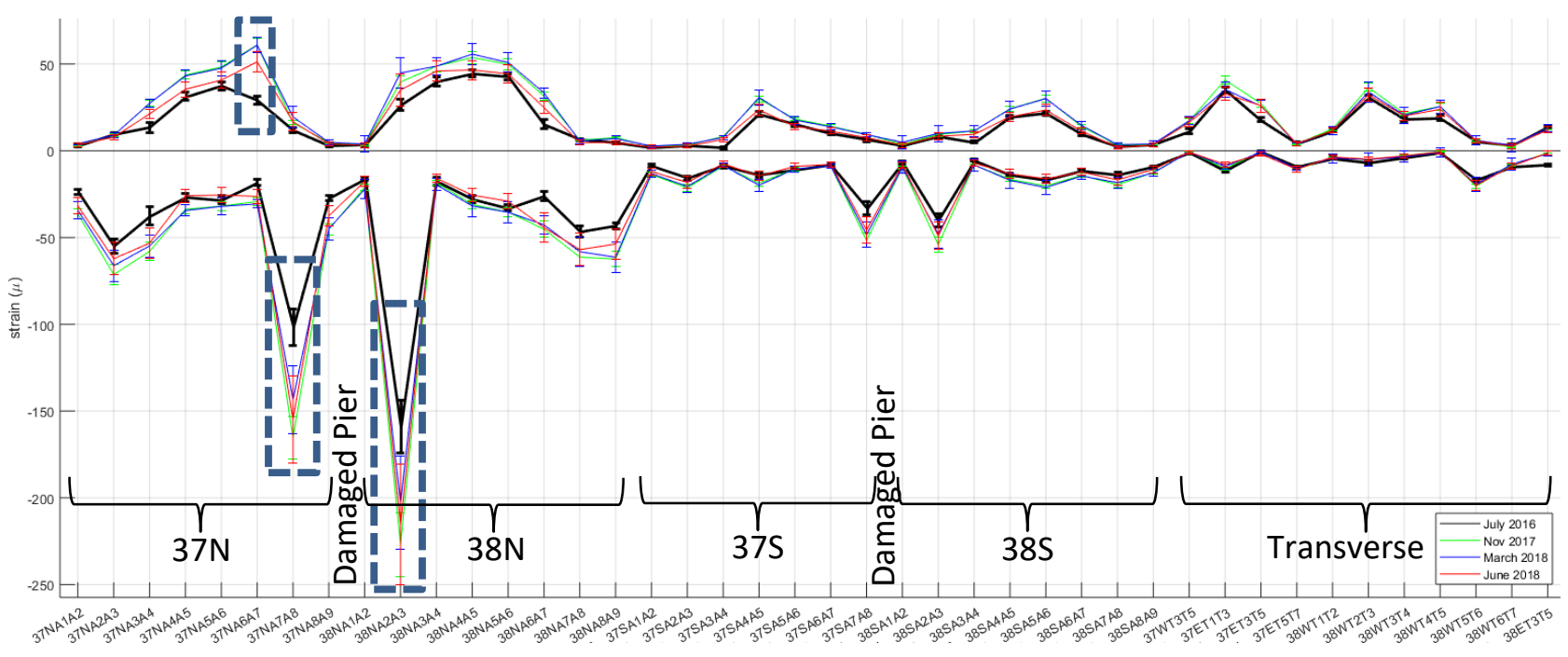

Figure 7. Mean value and standard deviation of the maximum and minimum peaks of FBG signals (see Figure 6) for 42 sensors underneath Arches 37 and 38, for the Class 185 3-carriege train that is heading East, exiting Leeds Station 


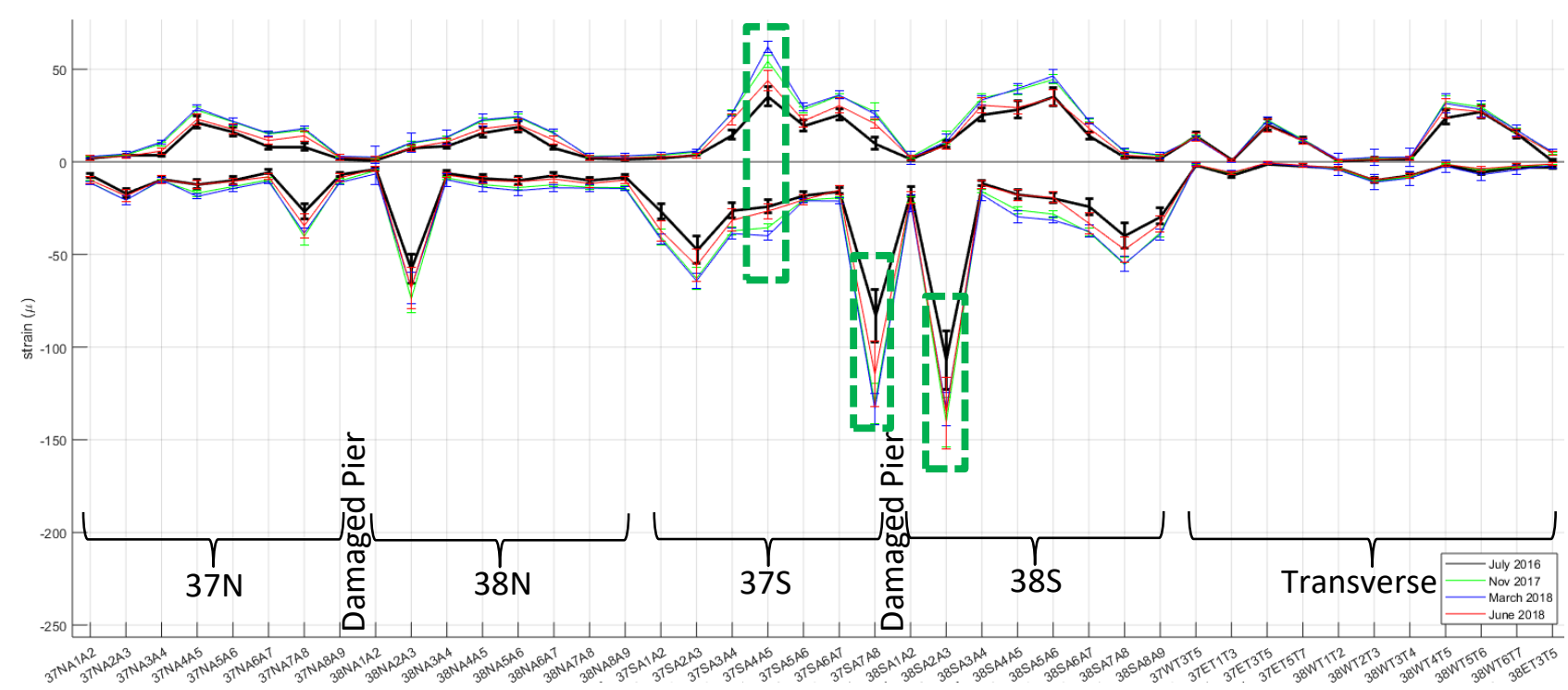

Figure 8. Mean value and standard deviation of the maximum and minimum peaks of FBG signals (see Figure 6) for 42 sensors underneath Arches 37 and 38, for the Class 185 3-carriege train that is heading West, entering Leeds Station.

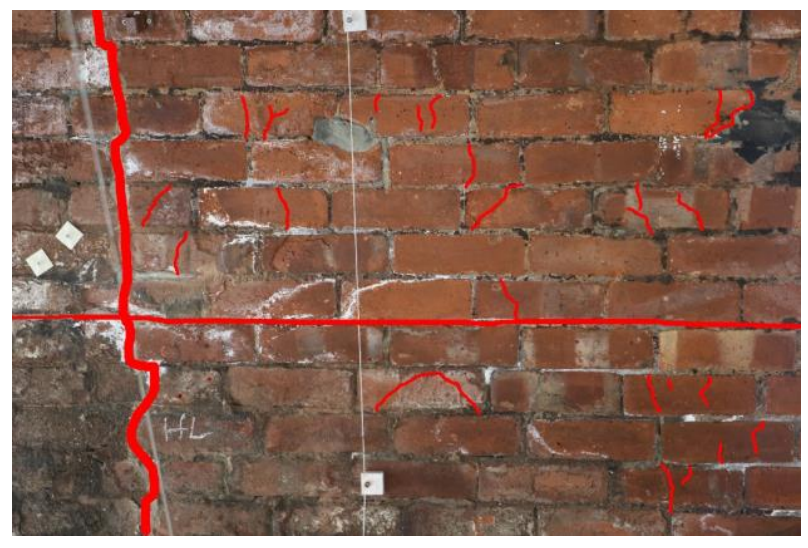

Figure 9. Cracks observed near sensor 37NA7A8

Figure 8 presents the same results for the Class 185 train, this time heading West, which explains why the south longitudinal sensors, 37S and 38S recorded bigger strains compared to the north longitudinal sensors, $37 \mathrm{~N}$ and $38 \mathrm{~N}$. Again, similar anomalies are observed for the sensors 37SA7A8 and 38SA2A3 next to the damaged pier shown inside the green dashed boxes in Figures 3 and 8 . At these locations, the increase of the dynamic deformation is again $50 \%$. Furthermore, both the tensile and compressive relative strain in the south keystone sensor 37SA4A5 at the most damaged arch (Arch 37) appears significantly increased.

Statistical analysis for the second most common train, Class 155 with two carriages (not shown here), presented identical results.
These results are matching with the formation of new micro-cracking in bricks, as shown in Figure 9. Although micro-cracking in bricks is observed in various locations in the bridge, the intensity is much larger near the sensors that presented the biggest increase of the compressive relative strain, such as the sensor 37NA7A8 presented in Figure 9.

\section{Conclusions}

The study presents a SHM monitoring system with the use of a FBG network to assess long term the performance of an ageing railway bridge. The system records the dynamic deformation of the bridge at 68 location for every train passage. Through the combination of signal processing techniques and statistical analysis it is possible to monitor changes in strain distribution over time, which are in excellent agreement with the observed damages. Furthermore, due to the high resolution of the sensors, these changes can be detected at a very early stage.

The permanent FO system proved to be resilient over the last year that it is in use. Long-term monitoring, combined with structural analysis modelling will further assess the effectiveness of the 2015 retrofitting intervention, and can be the base for the development of real-time streaming structural alert systems for ageing railway bridges. 


\section{Acknowledgements}

This work is being funded by the Lloyd's Register Foundation, EPSRC and Innovate UK through the Data-Centric Engineering programme of the Alan Turing Institute and through the Cambridge Centre for Smart Infrastructure and Construction (CSIC). Funding for the monitoring installation was provided by EPSRC under the Ref. EP/N021614/1 grant and by Innovate UK under the Ref. 920035 grant. The authors are grateful to Network Rail for providing power and technical support and for being able to utilize its infrastructure and monitoring data for this research.

\section{References}

[1] Ye C., Acikgoz S., Pendrigh S., Riley E., and DeJong M.J. Mapping deformations and inferring movements of masonry arch bridges using point cloud data. Engineering Structures. 2018; 173(15): 530-545.

[2] Orbán Z., and Gutermann M. Assessment of masonry arch railway bridges using nondestructive in-situ testing methods, Engineering Structures. 2009; 31(10): 22872298.

[3] Roca P., Cervera M., Gariup G., and Pela' L. Structural analysis of masonry historical constructions. Classical and advanced approaches. Archives of Computational Methods in Engineering. 2010; 17(3): 299325.

[4] Lau F.D.H., Butler L.J., Adams N.M., Elshafie M.Z.E.B., and Girolami M.A. Real-time statistical modelling of data generated from self-sensing bridges. Proceedings of the Institution of Civil Engineers - Smart Infrastructure and Construction. 2018; 171(1): 3-13.

[5] Lau F.D.H., Adams N.M., Girolami M.A., Butler L.J., and Elshafie M.Z.E.B. The role of statistics in data-centric engineering. Statistics \& Probability Letters. 2018; 136: 58-62.

[6] Hoole K. A Regional History of the Railways of Great Britain, Volume 4: The North East. Newton Abbot, David and Charles; 1973.
[7] Acikgoz S., DeJong M.J., Kechavarzi C., and Soga K. Dynamic response of a damaged masonry rail viaduct: Measurement and interpretation. Engineering Structures. 2018; 168: 544-558.

[8] Ferdinand P. The evolution of optical fiber sensors technologies during the 35 last years and their applications in structure health monitoring. 7th European Workshop on SHM, July 8-11, La Cité, Nantes, France; 2014.

[9] Glisic B., and Inaudi D. Fibre optic methods for structural health monitoring. England: John Wiley \& Sons; 2008.

[10] Kechavarzi C., Soga K., de Battista N., Pelecanos L., Elshafie M., and Mair R. Distributed Fibre Optic Strain Sensing for Monitoring Civil Infrastructure. London: ICE Publishing; 2016.

[11] Barrias A., Casas J.R., and Villalba S.A. Review of Distributed Optical Fiber Sensors for Civil Engineering Applications. Sensors. 2016; 16(5), 748. 